\title{
Magnetic Field Effects on the Plume of a Diverging Cusped-Field Thruster
}

\author{
Taylor Matlock, ${ }^{*}$ Stephen Gildea, ${ }^{*}$ Fuzhou Hu, ${ }^{\dagger}$ Nicholas Becker,${ }^{\ddagger}$ Paulo Lozano ${ }^{\S}$ \\ and Manuel Martínez-Sánchez \\ Massachusetts Institute of Technology, 77 Massachusetts Avenue, Cambridge MA, 02139, USA
}

The Diverging Cusped-Field Thruster (DCFT) uses three permanent ring magnets of alternating polarity to create a unique magnetic topology intended to reduce plasma losses to the discharge chamber surfaces. The magnetic field strength within the DCFT discharge chamber (up to $4 \mathrm{kG}$ on axis) is much higher than in thrusters of similar geometry, which is believed to be a driving factor in the high measured anode efficiencies. The field strength in the near plume region is large as well, which may bear on the high beam divergences measured, with peaks in ion current found at angles of around 30-35 from the thruster axis. Characterization of the DCFT has heretofore involved only one magnetic topology. It is then the purpose of this study to investigate changes to the near-field plume caused by altering the shape and strength of the magnetic field. A thick magnetic collar, encircling the thruster body, is used to lower the field strength outside of the discharge chamber and thus lessen any effects caused by the external field. Changes in the thruster plume with field topology are monitored by the use of normal Langmuir and emissive probes interrogating the near-field plasma. Results are related to other observations that suggest a unified conceptual framework for the important near-exit region of the thruster.

\section{Nomenclature}

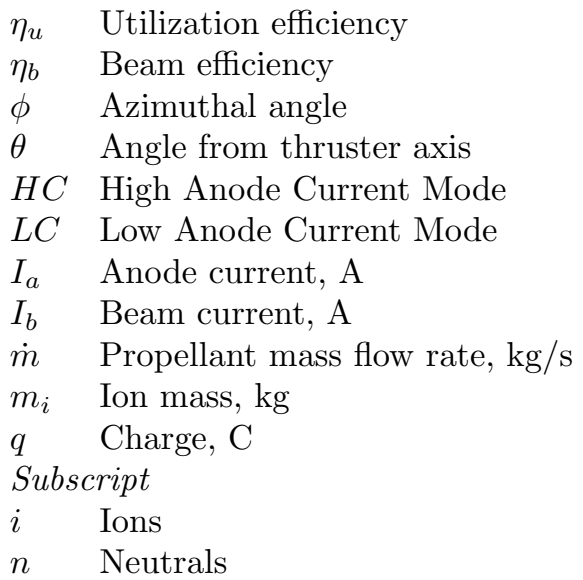

${ }^{*}$ Graduate Student, AIAA Student Member.

${ }^{\dagger}$ Undergraduate Student.

${ }^{\ddagger}$ Graduate Student, AIAA Student Member, KAUST Fellow.

$\S$ Professor, Aeronautics and Astronautics, Senior AIAA Member. 


\section{Introduction}

The miniaturization of plasma propulsion devices to low powers leads to the necessity to limit plasma losses to thruster surfaces through novel geometries in order to obtain acceptable thruster efficiencies. The Diverging Cusped-Field Thruster (DCFT) has a design inspired by CHT and HEMP thrusters which relies on strong magnetic fields to confine electrons away from thruster surfaces and promote ionization of an input neutral Xenon propellant. ${ }^{1-3}$ The concept has demonstrated high efficiencies at low power, but is hampered by a highly divergent plume structure, with the majority of the ion current coalescing into a conical shell with a half angle of more than $30^{\circ} \cdot{ }^{4-6}$ Visual evidence of this is shown in Figure 1.

A permanent magnet version of Princeton's CHT also displayed a hollow, highly divergent ion current profile, an effect which was hypothesized to be due to the higher magnetic field strength found external to the discharge chamber when using permanent instead of electro-magnetic circuits. ${ }^{10}$ LIF measurements of the electromagnet version of the CHT revealed strong radial electric fields near the thruster exit which lead to higher divergence angles when the magnetic field strength was increased. ${ }^{11}$ The magnetic field topology just downstream of the discharge chamber exit is known to have a large effect on the beam profile of regular geometry Hall thrusters as well, where the magnetic field lines are nearly plasma equipotentials and are used to great effect as ion lenses. ${ }^{12}$

It was originally postulated that the majority of the potential drop in the DCFT would occur within the discharge chamber, stepping down from one cusp to the next, thus making the shape of the magnetic field in the near plume relatively unimportant. Recent PIC modeling of our thruster along with data from a cylindrical DCF variant developed at Stanford, however, have shown the peak axial electric field occurring somewhat downstream of the thruster exit. ${ }^{13,14}$ The shape of the electric field, and thus the resulting plasma plume, is then expected to have a large dependence on the near-exit magnetic topology of the DCFT as has been found in similar plasma devices.

The simplest way to change the magnetic field in the plume of our permanent magnet device is by the addition of large pieces of magnetic materials to attenuate and redirect the field. This paper describes the results of the first such magnetic alteration attempted. The following section describes facilities and diagnostics used to characterize the changes in plume properties as well as the results of simulations and measurements of the altered magnetic field. Results are presented which map out the potential field and axial ion current profiles within the near plume of both the new and unaltered thruster. Conclusions are drawn as to the effects that this particular type of modification has on the plume structure, and a new conceptual model is discussed for the structure of the near-exit plasma.

\section{Experimental Apparatus}

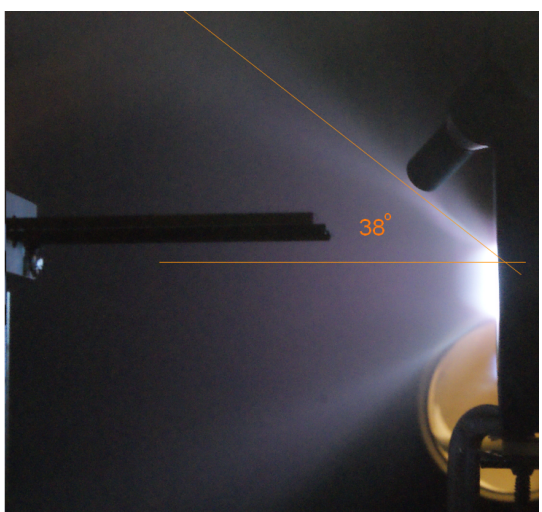

Figure 1. Image of the Collared DCFT operating in low current mode with cold Emissive probes.
Experiments were performed inside the MIT Space Propulsion Laboratory ASTROVAC vacuum chamber. The chamber is roughly 1.5 by $1.6 \mathrm{~m}$ with a pumping speed of roughly $7500 \mathrm{~L} / \mathrm{s}$ on Argon obtained using two CTI-Cryogenics cryopumps (CT10 and OB400). Pressure in the chamber is monitored using a cold cathode gauge with a calibration factor of 2.9 for Xenon. A Busek Inc. cathode is used as an electron source to initiate the discharge and neutralize the resulting ion beam.

The thruster body is left electrically floating, while the anode and cathode keeper potentials were applied with respect to the common chamber and facility ground. The anode voltage and keeper current are set by a pair of Agilent N5772A Programmable Power Supplies. Flow control and measurement to the anode and cathode is

provided by two Omega FMA-A2400 series mass flow controllers, allowing 0-10 $\mathrm{mL} / \mathrm{min}$ flows calibrated for Xenon. The cathode is run at $0.5 \mathrm{~A}$ on $0.2 \mathrm{mg} / \mathrm{s}$ of Xenon unless otherwise noted.

The thruster operates in two modes: a high current mode with strong anode current oscillations around $1-5 \mathrm{kHz}$, and a low current quiescent mode. The high current (HC) mode is a bifurcation marked by a steep jump in anode current as either the mass flow is increased or the anode potential is turned down, resulting 
in a visually diffuse plume. The low current (LC) mode, pictured in Figure 1, has a visually distinct plume with the apparent coalescence of the ion beam to an angle of around $30-40^{\circ}$ from the thruster centerline.

Probes are positioned within the chamber by a stack of two linear translation stages forming an $\mathrm{X}-\mathrm{Z}$ system driven by a pair of stepper motors. The $\mathrm{X}$ stage positions the probes to a certain radial distance from the thruster centerline and has a total travel length of 30in. The 40in-long $\mathrm{Z}$ stage controls the axial position of the probe with the face of the aluminum cap bolted to the thruster exit taken as $Z=0$. The step resolution of both stages is $5 \mu \mathrm{m}$. Motor control is automated within LabView and accomplished through a Velmex Inc. VXM motor controller capable of driving up to three stages.

Two to three emissive probes are installed on the stages for each experiment along with a planar Langmuir probe although only one emissive probe is connected to the associated circuitry at a given time. The extra probes create an added source of perturbation to thruster operation and plasma composition, but the short lifetime of the filament in a standard probe made redundancy quite beneficial. The probes are spaced radially by $2.22 \mathrm{~cm}$ with the collection surface of each at the same axial position; A schematic of the probe setup is shown in Figure 3. All probes lie radially along the vertical center of the thruster with a positional accuracy of $0.8 \mathrm{~mm}$. The radial and axial positions are estimated to be accurate within $0.5 \mathrm{~mm}$.

\section{II.A. Emissive probes}

Emissive Langmuir probes have found wide use as a tool for accurate plasma potential measurement in plasmas with low enough densities to allow for survival of the thin heated filament, due to their simple and robust principles of operation. ${ }^{7,8,14}$ The probe consists of a low work function emitter material which is heated to high enough temperatures to produce a thermionic electron emission current comparable to the ion current that can be drawn from the plasma. When the probe is biased highly negative compared to the local plasma potential the filament will collect ions from the plasma and emit a thermionic electron current, creating an increasingly large apparent ion current as the heater current is increased. As the probe tip potential approaches the plasma potential the probe will draw an electron current from the plasma while

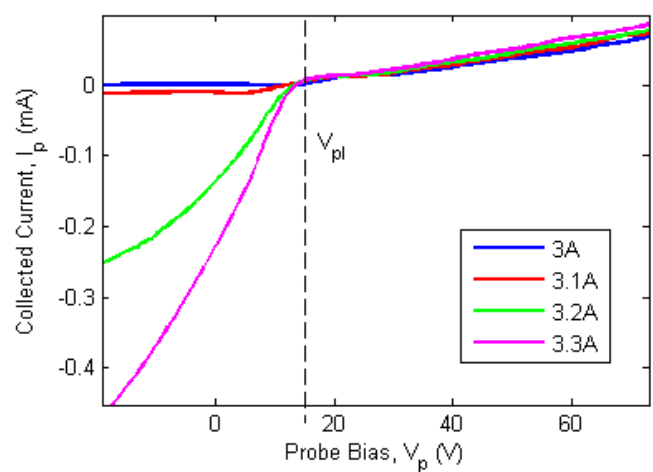

Figure 2. Emissive probe I-V traces at different levels of applied heating current. emitting electrons in the space charge limited regime. When the probe is at or above the local plasma potential the emitted electrons will be recollected by the probe and the probe current will collapse towards the normal curve collected by a cold, non-emitting, probe. The plasma potential can then be easily identified as the point where I-V curves taken with different levels of thermionic emission collapse. An example of this is shown in Figure 2 which shows I-V curves taken by one of the probes described here at several values of heater current applied to the filament.

It is also apparent from Figure 2 that the floating potential approaches the plasma potential as electron emission increases. Measuring the floating potential of a highly emissive probe is a plasma potential indication technique utilized by many due to its minimization of probe perturbations on the plasma as the probe draws no current. The floating potential using this method still sits below the true plasma potential by an amount on the order of the electron temperature. Even with complementary temperature data the exact shift can change dramatically with local magnetic field properties. ${ }^{8}$ We use the intersection of cold and hot I-V traces to determine plasma potential whenever possible, due to the strong, divergent magnetic fields found near the exit of our thruster, as this method is relatively

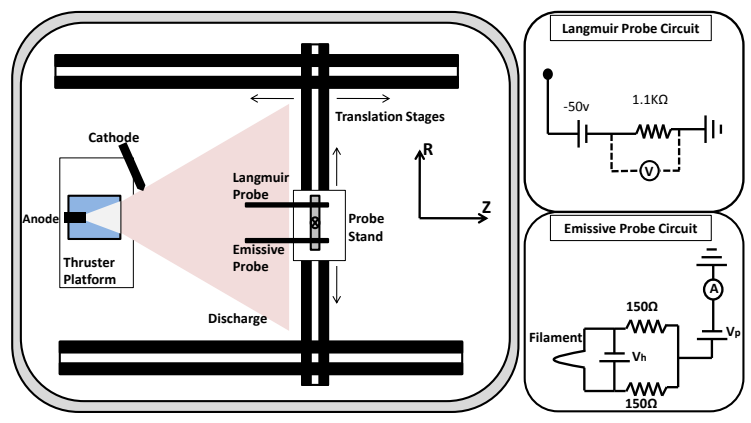
insensitive to B field effects.

The emissive probes used here are constructed from 0.125 in outer diameter, double bore alumina rods 
fit with stainless steel wire leads connected to $0.125 \mathrm{~mm}$ diameter $1 \%$ thoriated tungsten wire. The radius of curvature of the tungsten filament is roughly $1 \mathrm{~mm}$ on all probes. The bored out alumina is covered in the front of the probe using a castable alumina putty. Connections of the steel leads to the tungsten filaments were made a variety of ways, the least effective of which was by pressure fitting the bores with extra tungsten. More reliable connections were made by either crimping the filaments to nickel leads which were then soldered to the stainless wire or by directly spot welding tungsten to steel.

The tungsten filament was heated to thermionic emission by an Agilent E3634A DC power supply capable of floating up to $240 \mathrm{~V}$. The bias voltage was applied to the center of the probe by connecting a Keithley 2410 to a voltage divider with $150 \Omega$ legs on either side of the filament. The Keithley 2410 was also used as an ammeter to monitor current collected by the probe.

\section{II.B. Langmuir probe}

A planar Langmuir probe was also implemented for more reliable ion current measurements and as a back-up for the emissive probes. The I-V curves obtained from the Langmuir probe can be analyzed in the usual manner to yield measures of the electron temperature, ion saturation current and plasma potential. ${ }^{9}$ Since the probe is not mounted on a rotational stage, it only collects the axial component of the incoming ion flux, which is the only component providing thrust. Spatial profiles of the axial ion flux can yield information on the efficiency with which the thruster converts input neutral gas flow to usable ion flow in the plume as well as how much of the current being pumped through the anode power supply is actually contributing to the ion beam, using Equations 1-3, where $I_{b}$ can be used to estimate the $\dot{m}_{i}$ used in Equation 2 by assuming an entirely singly charged ion population exists in the plume.

$$
\begin{gathered}
I_{b}=\int_{0}^{2 \pi} \int_{0}^{R} J_{i z}(r) d r r d \phi \\
\eta_{u}=\frac{\dot{m}_{i}}{\dot{m}}=\frac{m_{i} I_{b}}{q \dot{m}} \\
\eta_{b}=\frac{I_{b}}{I_{a}}
\end{gathered}
$$

The Langmuir probe used here was made from 1/16th inch diameter tungsten rod inserted and set flush with a single bore $1 / 8$ th in diameter alumina tube. The length of the probe was set to match that of the longer emissive probes. The probe was set to a constant bias of $-50 \mathrm{~V}$ with respect to the vacuum chamber by a Kepco BOP 200-1D bipolar DC power supply and currents were read through a $1.1 \mathrm{k} \Omega$ resistor by a Fluke 77 IV voltmeter. An accuracy of $\pm 0.2 \mu \mathrm{A}$ was obtained.

\section{II.C. Magnetic Field}

The baseline thruster magnetic field circuit has been described thoroughly in previous work. ${ }^{4,6}$ The applied field is provided by three coaxial SmCo permanent magnets of alternating polarity, creating a multi-cusp field (3 radial and 4 axial) shown in Figure 4(a). The magnetic field outside of the thruster is not attenuated in any way in the original configuration, leading to fields as strong as $100 \mathrm{G}$ at a radius of up to $60 \mathrm{~mm}$ near the thruster exit where the field lines begin to turn upstream.

By attaching a thick magnetic collar around the thruster, the field strength external to the discharge chamber can be easily reduced. Such a configuration, modeled in Figure 4(b), also leads to field lines which diverge away from the thruster axis closer to the exit plane than in the baseline model. A 50G field is present at $\langle R, Z\rangle=<76 \mathrm{~mm}, 0 \mathrm{~mm}>$ in the baseline model, while the field is only $12 \mathrm{G}$ at that point with the collar added.

Figure 4 illustrates some important characteristics of the DCF magnetic configuration that have not so far received sufficient attention. The main point is the existence of a separatrix B-line roughly in front of each of the magnetic cusps. A separatrix is the line that separates the B-lines that bend upstream at the axis of symmetry from those that bend downstream. Up to the last cusp, these lines divide the domain into 'magnetic cells' with re-entrant lines; beyond the last separatrix, B-lines are free to expand into the plume region, and at least those that are close to the separatrix start in a paraxial direction, although all of them eventually loop back to the upstream direction. The cathode is typically on one of the B-lines that is not near the separatrix, although, given the line convergence towards the last cusp, its thruster-side end is not far from it. The plotting routine used in Figure 4 highlights these special lines and cells, in part by being 


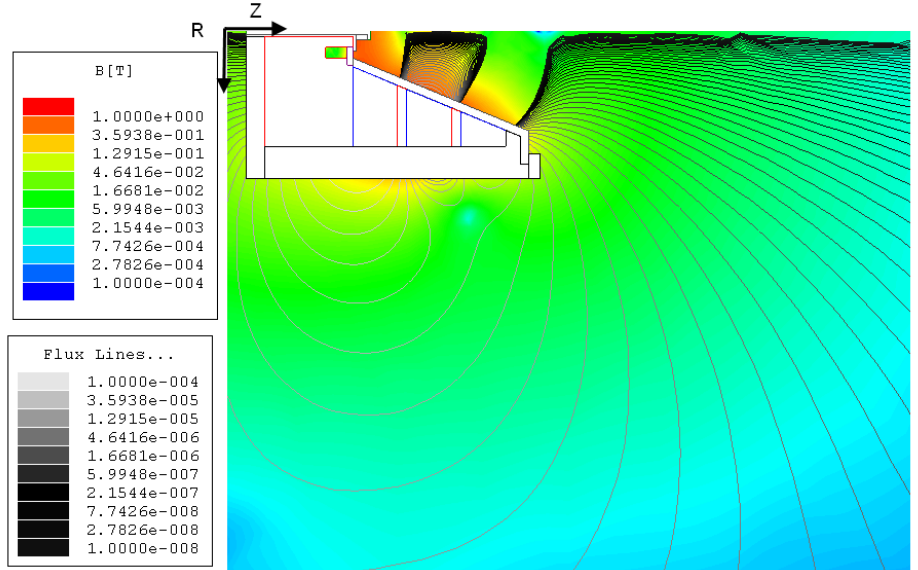

(a)

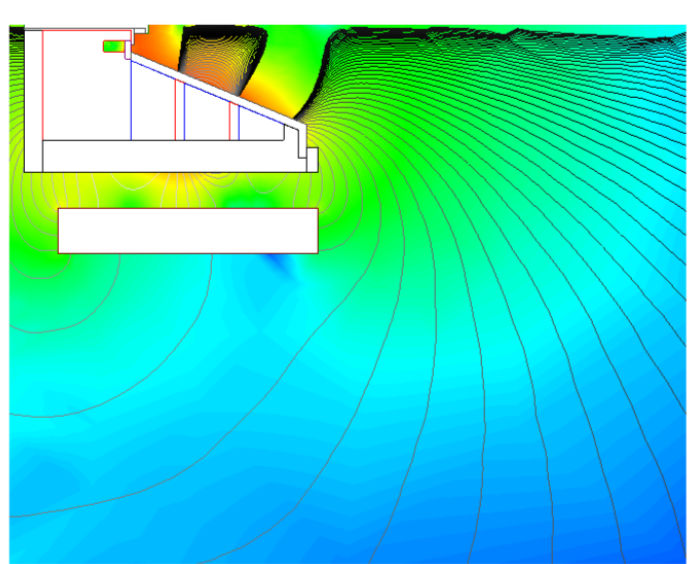

(b)

Figure 4. Ansoft Maxwell Simulations of magnetic field strength and flux lines for (a) Baseline (b) Collared configurations

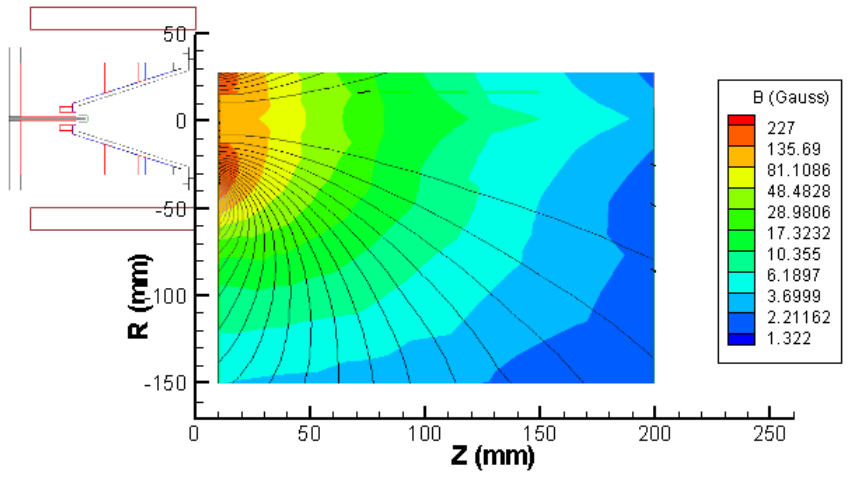

(a)

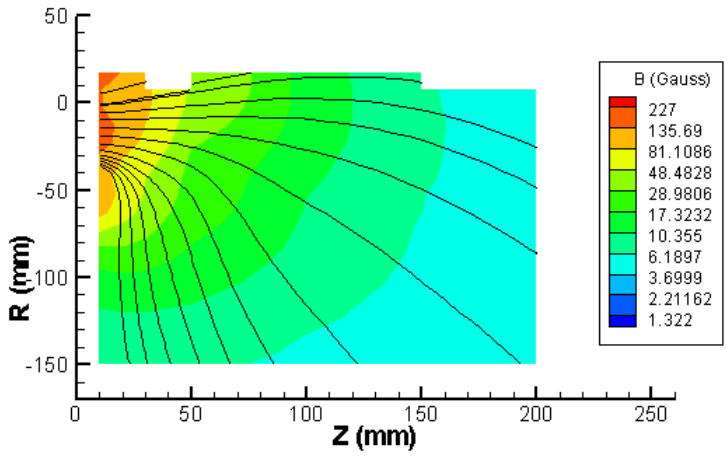

(b)

Figure 5. Magnetic field strength with overlaid flux lines from (a) Ansoft Maxwell SV simulations (b) DC Gaussmeter measurements.

unable to draw magnetic lines in alternate cells. We will return to these special geometrical features in our discussion of results. One fact that is already apparent at this point is that, despite large changes in B in the outside region, the separatrix line is barely affected by the magnetic collar (compare Figures $4 \mathrm{a}$ and $4 \mathrm{~b}$ ).

The collar itself is made from 0.5in thick iron tubing with an outer diameter of 5in and a length of 2.8in. Measurements of the field in the plume of the collared thruster, made with an AlphaLabs Inc. DC Gaussmeter, agree reasonably well with simulations of the field using Ansoft Maxwell SV. Simulations and measurements are shown in Figures 5(a) and (b) respectively. The main difference between the two is a dip in the measured field strength which occurs in the gap between the thruster outer diameter and collar inner diameter.

\section{Results}

Baseline testing was conducted at an anode potential of $400 \mathrm{~V}$ and a Xe mass flow of $0.49 \mathrm{mg} / \mathrm{s}$ resulting in a $160 \mathrm{~mA}$ LC mode. Plasma potential measurements shown in Figure 6 reveal a triply peaked profile along the radial direction. A diverging conical beam of high plasma potential emanates at a half angle of around $\theta=30^{\circ}\left(\theta=\tan ^{-1}(R / Z)\right)$, with a faster decaying peak running along the axis. The thruster began to exhibit erratic behavior as the emissive probes were brought within several centimeters of the exit plane, with large swings in anode current and brief changes in operational mode. The potential structure in the plume does not appear to coincide much with the applied magnetic field lines.

The addition of the iron collar impacted the ability to obtain stable LC modes over a wide range of conditions. Strong LC operation is achieved in the baseline thruster by lowering the anode flow rate or 
increasing either the anode voltage or keeper current. In the case of the collared thruster a low flow rate of

$0.49 \mathrm{mg} / \mathrm{s}$ and a keeper current of $1.5 \mathrm{~A}$ were both necessary to remove any flickering between modes. The emissive probe measured plasma potentials in this mode are shown in Figure 7, along with electric field vectors found by numerical differentiation of the data and an overlay of the simulated magnetic flux lines. The electric field vectors are somewhat rough due to the relative coarseness of the radial scans which were done in $1 \mathrm{~cm}$ increments everywhere in the plume, but they demonstrate the substantial divergence of the accelerating fields acting on ions which do not exit along the thruster axis.

The results in both magnetic configurations bear quite a few similarities with emissive probe measurements made on a cylindrical variant of the thruster, including the appearance of a potential valley along the thruster axis, just downstream of the exit. ${ }^{14}$ In the collared case, the plasma potential drops from $160 \mathrm{~V}$ at $5 \mathrm{~mm}$ from the exit to $16 \mathrm{~V}$ at $3 \mathrm{~cm}$, then rises toward $20 \mathrm{~V}$ at $5 \mathrm{~cm}$ before approaching $11 \mathrm{~V}$ further downstream.

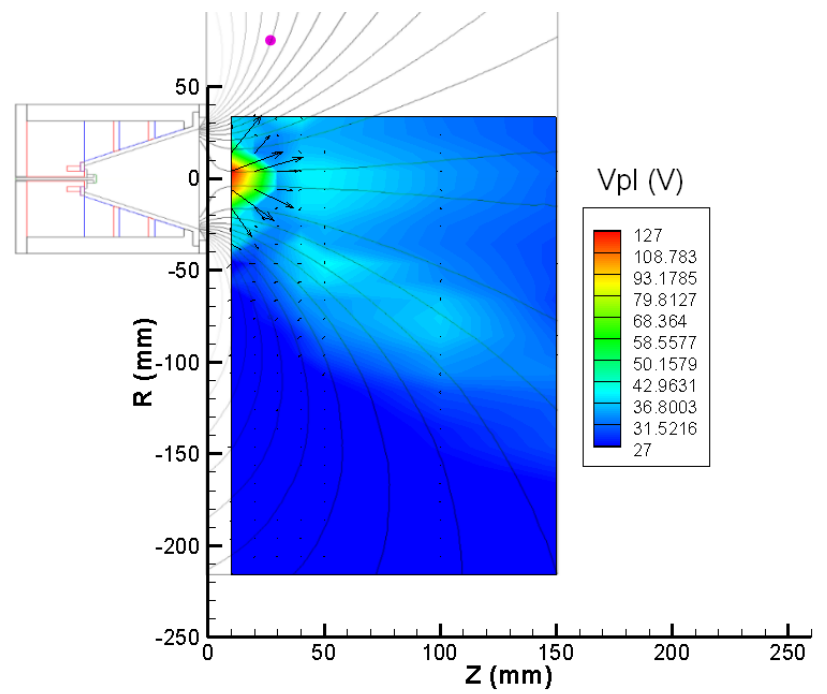

Figure 6. Plasma potential measured by emissive probe for baseline DCFT anode operating at 400V $160 \mathrm{~mA}$ and $5 \mathrm{sccm}$ of $\mathrm{Xe}$ w/ 0.5A keeper current. Calculated electric field vectors are plotted with length relative to magnitude. Simulated B field lines are overlaid. Pink dot shows cathode position in B field.

There is also a dip in the potential around $3 \mathrm{~cm}$ downstream following a $\sim 44^{\circ}$ angle along the divergent plasma potential ridge.

The baseline case of Figure 6 is similar, dropping from $120 \mathrm{~V}$ at $\mathrm{Z}=1 \mathrm{~cm}$ to a minima at $3 \mathrm{~cm}$ of $35 \mathrm{~V}$, up to a maxima of $39 \mathrm{~V}$ at $5 \mathrm{~cm}$ then back down towards $31 \mathrm{~V}$ at $15 \mathrm{~cm}$. The main differences between the two magnetic configurations are the overall lower plasma potential in the collared thruster plume, by a nearly constant $40 \%$, and an increase in divergence of the potential hill by roughly $14^{\circ}$. The overall drop in potential in the collared case may be partially attributed to the $150 \%$ increase in keeper current needed to maintain stable operation, a possiblity which can easily be tested by running the baseline case again at high keeper current. Potential measurements made with the collared thruster running in $\mathrm{HC}$ mode with $0.5 \mathrm{~A}$ running through the keeper, which are presented later, show far-field plume voltages of around $12 \mathrm{~V}$ as well, suggesting that the decrease in the potential off-axis is effected more by the change in magnetic field than the cathode properties. The addition of the collar also appears to shift the acceleration upstream

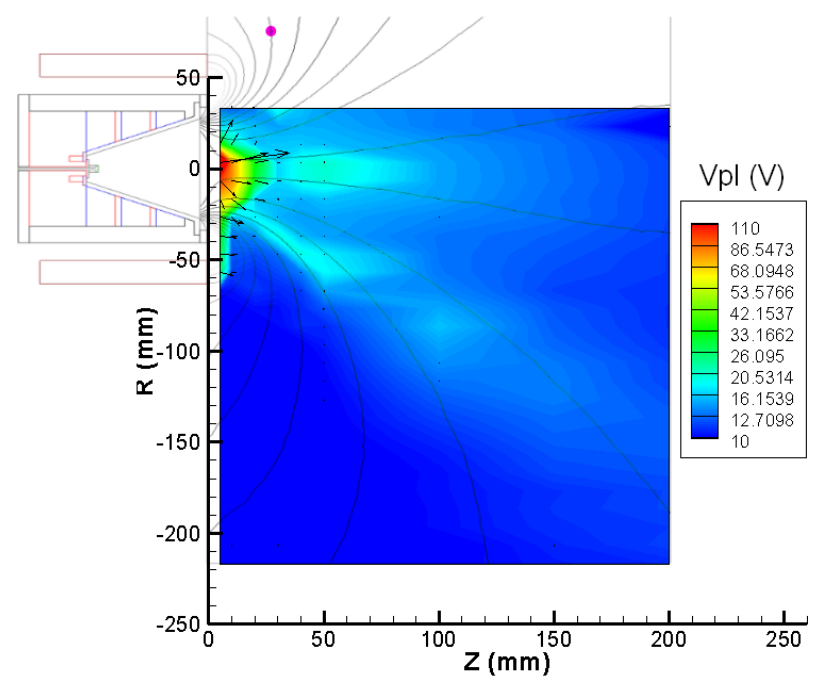

Figure 7. Plasma potential measured by emissive probe for collared DCFT anode operating at $400 \mathrm{~V} 160 \mathrm{~mA}$ and $5 \mathrm{sccm}$ of Xe w/ 1.5A keeper current. Calculated electric field vectors are plotted with length relative to magnitude. Simulated B field lines are overlaid. Pink dot shows cathode position in B field.

by $1 \mathrm{~cm}$, although baseline data were not obtainable less than $10 \mathrm{~mm}$ from the thruster exit.

Perhaps the clearest comparison between the two magnetic configurations comes from ion current measurements taken $20 \mathrm{~cm}$ from the exit face of the thruster, shown in Figure 8. The figure shows clearly that the peak in ion current shifts to a higher angle from the thruster axis when the iron collar is attached, changing from $29.7^{\circ}$ in the $\mathrm{HC}$ baseline $\left(0.73 \mathrm{mg} / \mathrm{s}\right.$ of Xe for a $560 \mathrm{~mA}$ anode current) to $33.4^{\circ}$ in the collared $\mathrm{HC}$ mode and from $33.4^{\circ}$ to $36.3^{\circ}$ in the base and collar LC modes respectively.

Conversely, Figure 8 appears to indicate a slight bump in the beam efficiency when the collar is attached. The measured ion beam current integrated up to a radius of $25 \mathrm{~cm}$ is $150 \pm 3 \%$ and $154 \pm 11 \%$ of the anode 
current for the baseline thruster in high and low current modes respectively while it is $166 \pm 8 \%$ (HC) and $159 \pm 11 \%$ (LC) for the collared variant. The actual beam efficiencies are of course less than unity, with the discrepancy expected to be due mainly to sheath expansion effects and collection of charge exchage ions at large radial distances.

Two-dimensional maps of the axially projected ion current measured by the Langmuir probe can be seen in Figures 9 and 10 for the baseline and collared thrusters respectively. It is clear from Figure 9 both that the axial ion current visits a local minima along the the thruster axis in the same location of the plasma potential valley, $3 \mathrm{~cm}$ downstream of the exit, and that the plume has detached from the magnetic field. The plasma potential is plotted over the ion current density of Figure 10 as black contour lines to further demonstrate the strong correlation between regions of high ion flux and high plasma potential found in both configurations. The peak ion current does not appear to emanate from the origin of our coordinate system in either configuration but instead from some region around 10 to

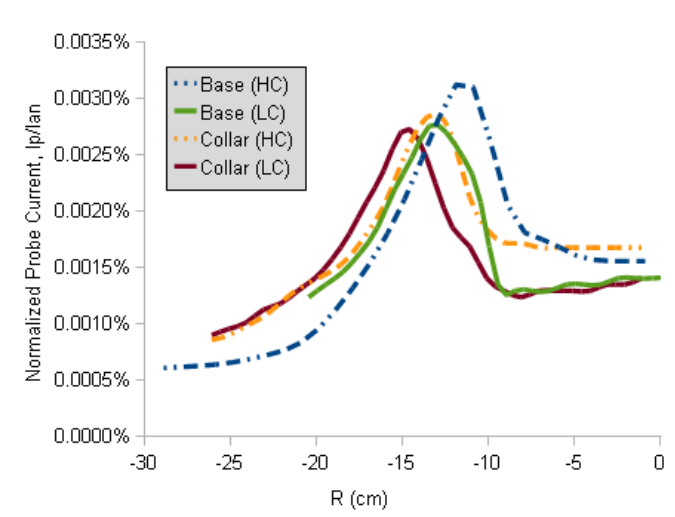

Figure 8. Axial ion current normalized by anode current vs radial distance from thruster axis for both baseline and collared DCFT operation at $\mathrm{Z}=20 \mathrm{~cm}$. $20 \mathrm{~mm}$ off axis.

\section{III.A. Discussion of LC mode results. Role of the magnetic separatrix}

As noted, the bright plasma shell appears to be anchored near the exit plane on a ring with radius about one half that of the thruster there. This is clearly visible in Figure 1. Figure 1 also shows a protruding luminous 'bulb', which reference to Figures 6 and 7 identifies with the rapid rise of potential as the exit plane is approached from downstream. Comparison to Figure 4 makes it very likely that the downstream edge of this 'bulb' is just slightly beyond the separatrix line associated with the last cusp. Although not shown here, photographs and visual observations from a 45 degree angle make this suggestion even more appealing, as the bulb is revealed to be really a thin luminous shell roughly coinciding with the separatrix, at least near the outer radius (the luminosity is weaker near the centerline). This luminosity, and the fact that the strong potential drop starts at this location, indicate strong ionization activity on and very near this portion of the separatrix.

The explanation for this localization of the ionization is now not difficult to understand. Electrons from the cathode initially fill the cathode-connected B surface, and execute repeated back and forth passes between the cathode and the sheath edge on the ceramic wall, perhaps being forced back earlier through magnetic mirroring. Occasional collisions, aided by some weak turbulent scattering, in the presence of the downstream directed E field, have the effect of gradually allowing these electrons to cross magnetic surfaces and approach the separatrix. Once this particular surface is crossed, the electrons switch to the next upstream magnetic cell and start to bounce back and forth between the two cusps. Notice that from the beginning of this new phase, these electrons reach the next upstream cusp not far from the next separatrix,

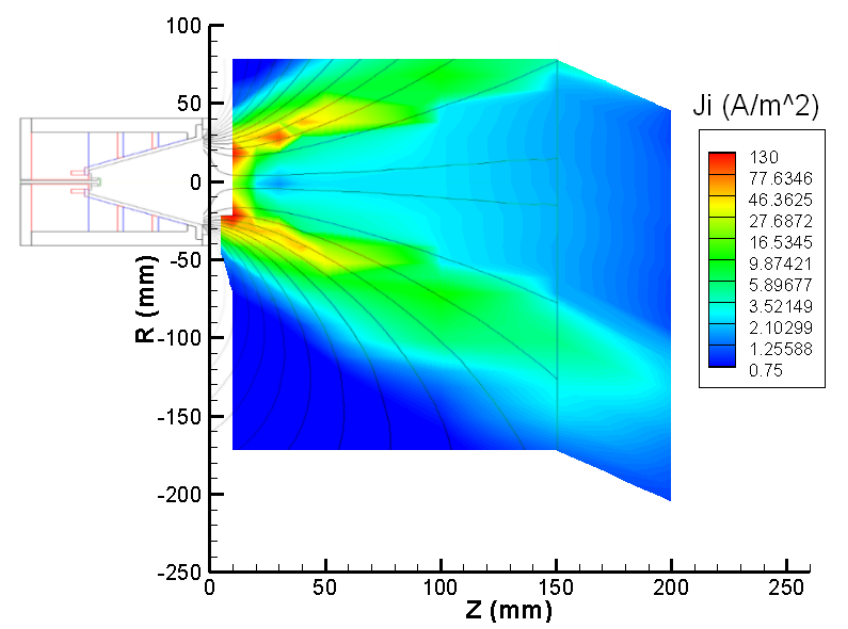

Figure 9. Axially directed ion current density for baseline DCFT anode operating at $400 \mathrm{~V} 160 \mathrm{~mA}$ and $5 \mathrm{sccm}$ of Xe. Simulated B field lines are overlaid. so it will not take many bounces to progress into a new upstream cell, and eventually into the anode. By contrast, the initial phase in which cathode electrons are electrostatically trapped between the vicinity of the downstream separatrix and some downstream point can be fairly long, depending on cathode location; 
since in each bounce the electrons will approach close to the outer edge of the separatrix, a region of intense ionization is to be expected there, as observed.

The mechanism just described serves to explain the 'hollow cone' structure of the plume and, to some extent, its angle. Ions originate, as explained, near the outer rim of the last separatrix, and ionization rate must fall steeply as one proceeds radially inwards on this surface; this is simply a reflection of electron continuity in the converging B field. The majority of ions will then start their acceleration roughly perpendicular to the separatrix, which, by reference to Figure 4, is about the observed angle for the luminous streak of Figure 1.

Two additional observations can be made: As noted in Section II, magnetic lines near the last separatrix tend to come close to the axis at some point downstream of the exit. This 'bottleneck' is then a second region of statistical electron concentration, and, given the presence of some neutrals, will give rise to some secondary ionization. This is very likely the origin of the slight potential maximum near the axis that was described in Section III (ions linger near their point of origin longer than electrons, giving rise to a characteristic potential bump in ionization regions). The second observation is that one does indeed expect some neutrals to be present near the axis, since chances of escape are greatest there. This is a weakness of the design, and explains the relatively low utilization fraction

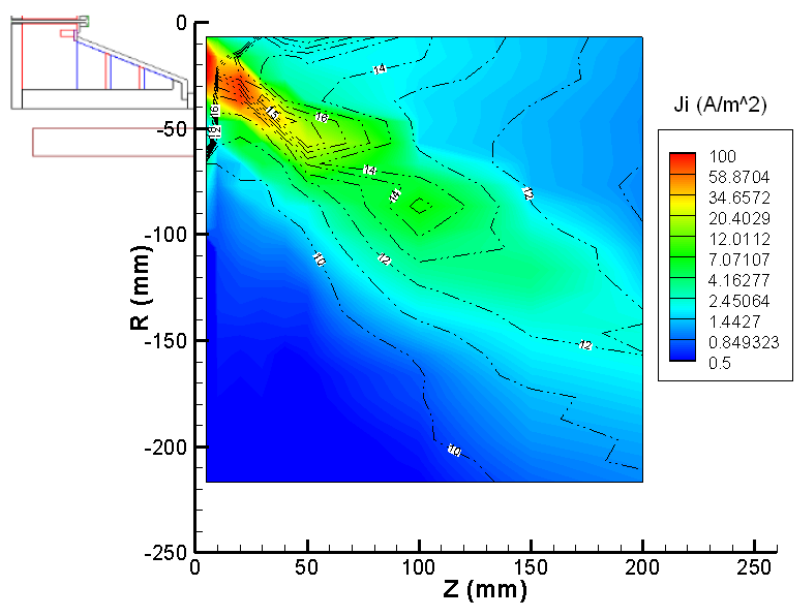

Figure 10. Axially directed ion current density for collared DCFT anode operating at $400 \mathrm{~V} 160 \mathrm{~mA}$ and $5 \mathrm{sccm}$ of Xe. Plasma potential contours overlaid from 10 to $20 \mathrm{~V}$ by $1 \mathrm{~V}$. of the propellant, although the net efficiency is reasonably high because of high current and voltage efficiencies. Perhaps this can be taken as an indication that a smaller divergence angle might improve performance.

Finally, the observation that electrons should have comparatively little trouble crossing the upstream separatrices implies that one should not expect much ionization to occur near the upstream cusps. It remains to be seen whether the magnetic bottle in front of the anode is sufficient to anchor strong ionization there; this may be the meaning of visual observations of strong luminosity in the anode region, especially in the High Current mode.

\section{III.B. HC Mode}

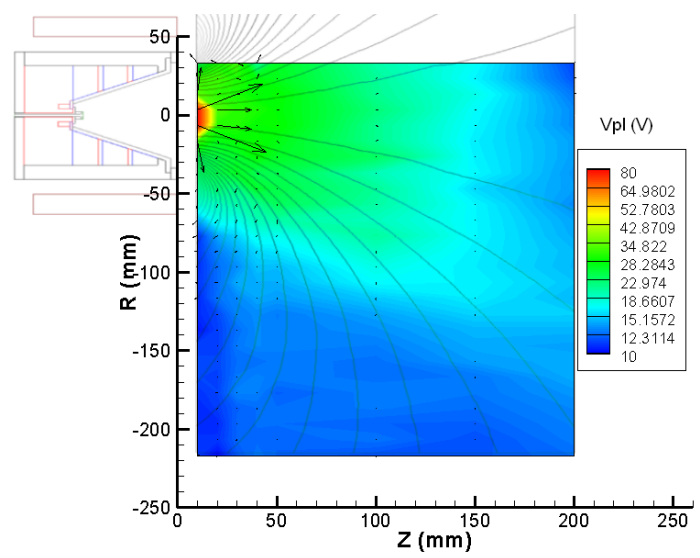

Figure 11. Plasma potential measured by emissive probe for collared DCFT anode operating at $400 \mathrm{~V}$ $220 \mathrm{~mA}$ and $5 \mathrm{sccm}$ of Xe. Calculated electric field vectors are plotted with length relative to magnitude. a Simulated B field lines are overlaid.
A stable $\mathrm{HC}$ mode was obtained in the collared configuration by decreasing the keeper current from $1.5 \mathrm{~A}$ to the normal $0.5 \mathrm{~A}$ used in all baseline testing. The anode potential and flow rate were kept at the values used for LC mode characterization $(400 \mathrm{~V}$ and $0.49 \mathrm{mg} / \mathrm{s})$ but the anode current increased to $220 \mathrm{~mA}$. The resulting plasma potential map can be seen in Figure 11. The shape of the plume potential contrasts with that found in LC mode in the same manner as the two plumes compare visually: the $\mathrm{HC}$ mode is diffuse and looks the same everywhere up to a certain angle while the LC mode is sharply focused with easily discernible features. The measured electric field is nearly perpendicular to the magnetic field lines only in the region near the thruster where the magnetic field lines turn upstream.

High spatial resolution scans were performed along the thruster axis in each of the two modes for further comparison and in an attempt to resolve the main acceleration 
mize probe dwell time in the plume. The plasma potential is taken as the maximum in the first derivative of the electron emitting I-V curves. Comparisons of this method with previously obtained measurements which find the intersection of hot and cold I-V traces showed discrepancies of usually less than $10 \mathrm{~V}$.

The results of axial scans in each mode are shown together in Figure 12 where the $250 \mathrm{~mA}$ data are in $\mathrm{HC}$ and the $150 \mathrm{~mA}$ data are in LC. The modes show very similar on-axis traits. Both retain roughly $72 \%$ of the applied voltage $5 \mathrm{~mm}$ downstream of the exit and reach a peak axial electric field between 10 and $12.5 \mathrm{~mm}$. The main difference is in the location and amplitude of the small potential valley near the exit. In $\mathrm{HC}$ mode the plasma reaches a local minimum of $41 \mathrm{~V}$ a distance of $22.5 \mathrm{~mm}$ from the exit before rising up to $56 \mathrm{~V}$ at $30 \mathrm{~mm}$ and heading back down towards $23 \mathrm{~V}$. In LC mode that minimum occurs much further downstream at around $34 \mathrm{~mm}$ and the voltages

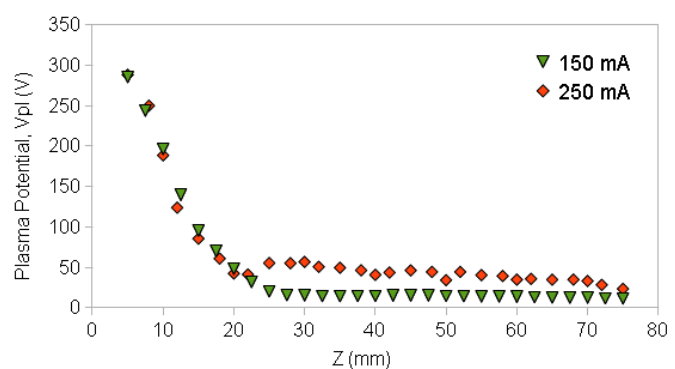

Figure 12. Plasma potential measured by emissive probe on axis for collared DCFT anode operating at $400 \mathrm{~V}$ and $5 \mathrm{sccm}$ of $\mathrm{Xe}$ for two values of anode current. are much lower, going from a low of $14.5 \mathrm{~V}$ up to $15.2 \mathrm{~V}$ at $47.5 \mathrm{~mm}$ then down to less than $12 \mathrm{~V}$.

The fact that the main potential drop profile is the same in both modes, despite the drastically different time signature of the currents (nearly constant in the LC mode, violently oscillatory in the HC mode) is not understood at this time. The possibility has been considered of alternative ignition at two cusps, but one would then expect to see very different potential profiles (even when time-averaged) past the last ionization region. Perhaps these differences are not to be seen near the axis, and this should be further investigated.

\section{Conclusions}

Electric field measurements in the near plume of a magnetic variant of the DCFT were made by numerically differentiating potential profiles obtained with an emissive probe technique. The fields exhibit large radial components near the thruster exit and in the wings of the plume where the diverging magnetic field has a stronger effect on the potential profile. The addition of an iron collar around the outside of the thruster served to reduce the magnetic field strength outside of the discharge chamber with the intent of lessening the radial electric fields set up there by diverging lines of magnetic flux. Contrarily, a slight increase in the ion beam divergence was observed. This may be due to the magnetic field lines peeling off from the axis closer to the thruster exit steering the electrons outward or from an increased radial electric field component at the acceleration region caused by the lower potentials found away from the axis. In either case the effect of the magnetic collar on the thruster efficiency appears to be negligible.

It is hypothesized that the seperatrix formed by the radial and axial cusps closest to the cathode plays a larger role in determining the shape of the electric field than does the shape or strength of the magnetic field further from the exit. Electrons from the cathode congregate at the radial cusp near the thruster exit, where the majority of the ion acceleration occurs, and thus the line central to the cusp where electron density is a maximum is likely to play an important role in the development of the electric field. Future experiments will focus on alteration to the shape of this separating line.

\section{Acknowledgments}

This work is funded by the U.S. Air Force Office of Scientific Research.

\section{References} 2006

${ }^{1}$ Raitses, Y., Smirnov, A., Fisch, N.J. Cylindrical Hall Thrusters, 37th AIAA Plasmadynamics and Lasers Conference,

${ }^{2}$ Smirnov, A., Raitses, Y., Fisch, N.J. Electron cross-field transport in a low power Cylindrical Hall Thruster, Physics of Plasmas, 2004.

${ }^{3}$ Kornfeld, G., Koch, N., Harmann, H. Physics and Evolution of HEMP-Thrusters, 30th International Electric Propulsion Conference, 2007.

${ }^{4}$ Courtney, D., Martinez-Sanchez, M.Diverging Cusped-Field Hall Thruster (DCHT), 30th International Electric Propulsion Conference, 2009.

${ }^{5}$ Courtney, D., Lozano, P., Martinez-Sanchez, M. Continued Investigation of Diverging Cusped Field Thruster, 44th AIAA/ASME/SAE/ASEE Joint Propulsion Conference, 2008. 
${ }^{6}$ Courtney, D., Development and Characterization of a Diverging Cusped Field Thruster and a Lanthanum Hexaboride Hollow Cathode,Masters Thesis, Massachusetts Institute of Technology, 2008.

${ }^{7}$ Ye, M., Takamura, S. Effect of space-charge limited emission on measurements of plasma potential using emissive probes, Physics of Plasmas, Vol.7, 2000.

${ }^{8}$ Fujita, H., Nowak, S., Hoegger, BA., Schneider, H. Potential measurements by an emissive probe in a magnetized plasma, Physics Letters, Vol.78A, 1980.

${ }^{9}$ Hutchinson, I.H Principles of Plasma Diagnostics, Cambridge University Press, 2002.

${ }^{10}$ Raitses, Y., Merino, E., Parker, J., Fisch, N. Operation and plume measurements of miniaturized cylindrical Hall thrusters with permanent magnets, 45th AIAA/ASME/SAE/ASEE Joint Propulsion Conference, 2009.

${ }^{11}$ MacDonald, N., Cappelli, M., Hargus, W. Laser-Induced flourescence velocity measurements of a low power cylindrical Hall thruster, 31st International Electric Propulsion Conference, 2009.

${ }^{12}$ Sommerville, J.D., King, L.B., Chiu, Y.H, Dressler, R.A. Ion-Collision Emission Excitation Cross Sections for Xenon Electric Thruster Plasmas, Journal of Propulsion and Power, 2008.

${ }^{13}$ Gildea, S., Martinez-Sanchez, M. Improvements in Divergent Cusped-Field Thruster Modeling, ESA/A3F Space Propulsion Conference, San Sebastián 2010.

${ }^{14}$ Young, C., Smith, A., Cappelli, M. Preliminary Characterization of a Diverging Cusped Field (DCF) Thruster, 31st International Electric Propulsion Conference, 2009.

10 of 10 